Fetal Diagnosis and Therapy
Fetal Diagn Ther 2019;46:285-295

DOI: $10.1159 / 000496202$
Received: August 30, 2018

Accepted after revision: December 13, 2018

Published online: March 12, 2019

\title{
Prenatal Diagnosis, Management, and Outcome of Fetal Subdural Haematoma: A Case Report and Systematic Review
}

\author{
Ka Wang Cheung ${ }^{a, b}$ Lee Na Tan ${ }^{a, c}$ Mimi Tin Yan Seto ${ }^{b}$ Shruti Moholkar $^{d}$ \\ Geraldine Masson $^{e}$ Mark D. Kilby ${ }^{a, f}$ \\ aThe Fetal Medicine Centre, Birmingham Women's and Children's Foundation Trust, Birmingham, UK; \\ ${ }^{b}$ Department of Obstetrics and Gynaecology, Queen Mary Hospital, The University of Hong Kong, \\ Hong Kong SAR, China; 'Department of Obstetrics and Gynaecology, Hospital Raja Permaisuri Bainun, Ipoh, Malaysia; \\ dDepartment of Radiology, Birmingham Women's and Children's NHS Foundation Trust, Birmingham, UK; \\ eDepartment of Obstetrics and Gynaecology, University Hospital of North Midlands, Stoke-on-Trent, UK; \\ ${ }^{f}$ The Institute of Metabolism and Systems Research, University of Birmingham, Birmingham, UK
}

\section{Keywords}

Intracranial haemorrhage $\cdot$ Haematoma Subdural haematoma · Pregnancy · Ultrasonography · Magnetic resonance imaging

\begin{abstract}
Background: Fetal subdural haematoma (SDH) is associated with poor prognosis. Objective: The conflicting evidence from the literature presents a challenge in prenatal counselling. We present a case study and systematic review of the literature for the management and outcome of fetal SDH. Methods: Systematic search of electronic database. Results: A total 45 cases were extracted from 39 papers. Prenatal ultrasonographic features were intracranial echogenicity $(42 \%)$, lateral ventriculomegaly (38\%), presence of an intracranial mass (31\%), macrocephaly (24\%), midline deviation of cerebral falx (20\%), and intracranial fluid collection (11\%). Further secondary features were noted including reversed diastolic flow in the middle cerebral artery $(11 \%)$, echogenic bowel (4\%), hydrops fetalis (2\%), and elevated middle cerebral artery peak systolic velocity (2\%) (all highly likely to be associated with fetal anaemia). The rates of termination of
\end{abstract}

pregnancy, stillbirth, and neonatal death were 18\% (8/45), $16 \%(7 / 45)$, and $11 \%(5 / 45)$, respectively. Overall, therefore, the fetal and perinatal mortality was $32 \%(12 / 37)$. Amongst the 24 survivors with available neurological outcome, $42 \%$ (10/24) and 58\% (14/24) had abnormal and normal neurological outcome, respectively. Underlying aetiology of fetal SDH was not identified in 47\% (21/45). Fetal SDH with an identifiable underlying aetiology was the only factor associated with a higher chance of normal neurological outcome when compared to fetal SDH without a detectable cause (78.5 vs. $21.4 \%, p=0.035$ ). Conclusions: Stillbirth and neonatal death occurred in a significant proportion of fetal SDH. $58 \%$ of survivors had normal neurological outcome, and better prognosis was seen in SDH with identifiable underlying aetiology.

(c) 2019 S. Karger AG, Basel

\section{Introduction}

Fetal intracranial haemorrhage may result in significant fetal and neonatal mortality and long-term neurological morbidity in surviving babies. The estimated inci-

\section{KARGER}

(c) 2019 S. Karger AG, Basel

E-Mail karger@karger.com

www.karger.com/fdt
Ka Wang Cheung

6/F, Professorial Block

Queen Mary Hospital, 102 Pokfulam Road

Hong Kong SAR (China)

E-Mail kelvincheung82@hotmail.com 
dence is $0.6-1 / 1,000[1-3]$ and this may be subclassified into intraventricular, intracerebral, cerebellar, subarachnoid, and subdural, depending on the anatomical location.

Subdural haematoma (SDH) is a blood collection outside the brain between the dura and arachnoid mater. It can occur in $8 \%$ of asymptomatic neonates after delivery without significant neurological consequences [4]. Advances in ultrasound imaging has increased prenatal detection of this entity, which is generally associated with poor prognosis. The overall adverse perinatal outcomes of fetal SDH, including stillbirth, neonatal death, and abnormal neurological outcome, is around $80 \%$, with the remaining $20 \%$ having normal neurological outcome [ 1 , $5,6]$. In a cohort series of 41 cases, Vergani et al. [1] reported the outcomes of fetuses and neonates as either having normal/mild neurological sequalae or severe neurological sequelae/death. They found severe neurological complications and fetal or neonatal death in $88 \%$ cases of fetal SDH (7/8), compared to $45 \%$ (9/20) with intraventricular and $92 \%(12 / 13)$ with parenchymal haemorrhage. The individual data on perinatal mortality and neurological deficit was not available. Similarly, a cohort study by Akman and Cracco [5] reported 59\% stillbirth (19/32), $16 \%$ neonatal/ infant death (5/32), 16\% neurological deficit (5/32), and $9 \%$ normal neurological outcome (3/32) in 32 cases of SDH. The mortality rate was $75 \%(24 / 32)$ and the rate of neurological deficit among survivors was $37.5 \%(3 / 8)$. However, a review by Ghi et al. [6] found only $12 \%$ of mortality rate $(2 / 17)$ and normal neurological outcome in $67 \%$ of survivors $(6 / 9)$ from SDH. The conflicting evidence from the literature presents a challenge in prenatal counselling. We hereby present a recent case study and perform a systematic review of the literature on prenatally detected fetal SDH.

\section{Case and Methods}

\section{Case}

A 25-year-old G2P0 was referred to our fetal medicine center at 27 weeks and 1 day of gestation for suspected intracranial haemorrhage. This was a consanguineous couple from Eritrea. Her past medical record was unremarkable with no personal or family history of bleeding disorder and there was no history of trauma.

Prenatal ultrasonography was performed at 27 weeks and 0 days of gestation at her local hospital when she presented with reduced fetal movement, which showed ventriculomegaly and suspected intracranial haemorrhage. A repeat ultrasonography in our fetal medicine centre the next day revealed a right-sided SDH with midline shift (Fig. 1a) and bilateral ventriculomegaly (ventricular width of the atrium $\sim 15 \mathrm{~mm}$ ) (Fig. 1b). There was reversed velo- cimetry during diastole in the left middle cerebral artery Doppler and the peak systolic velocity was increased at $76 \mathrm{~cm} / \mathrm{s}(2.2 \mathrm{MoM})$ (Fig. 1c), indicative of fetal anaemia. The amniotic fluid volume and the umbilical artery Doppler were normal. Intrauterine transfusion was deemed unsuitable in this circumstance as there was increased risk complications of performing an invasive procedure in a fetus with a possible existing bleeding tendency, and there was lack of evidence to support that the benefits of intrauterine transfusion outweigh the risks in fetuses with SDH. The significantly increased risks of stillbirth, neonatal death, and severe neurological disability as well as the option of termination of pregnancy were discussed. The couple decided at this point for continuation of pregnancy with expectant management and was planned for twice weekly ultrasonography.

In utero magnetic resonance imaging (iuMRI) of fetal brain was performed at 27 weeks and 2 days of gestation, which confirmed the ultrasonographic findings. The larger haemorrhage was seen over the right cerebral hemisphere and the smaller one over the left cerebral hemisphere, with significant midline shift to the left (Fig. 1d, e). In addition, there was intraventricular haemorrhage (Fig. 1f). These haemorrhages demonstrated intermediate to low T2 signal and intermediate T1 signal with slightly higher T1 signal along the margins of the compressed right cerebral hemisphere and laterally against the right side of the cranium. Additional extra-axial haematoma was present in the posterior fossa compressing and distorting the cerebellum and abutting the inferiorly displaced right temporal horn. Cerebellar herniation into the foramen magnum was noted. Bilateral cerebral infarction in most of the right cerebral hemisphere (sparing the inferior right frontal lobe) and left cerebral hemisphere with porencephaly in the left frontal lobe were seen on all conventional sequences and diffusion-weighted imaging (Fig. 1g, h). Maternal serology did not suggest recent maternal viral infections (toxoplasma, parvovirus, and cytomegalovirus), nor the presence of fetal alloimmune thrombocytopenia (parental human platelet antigen genotyping negative for 1a, 2a, 3a, 4a, 5a, 6a, 9a, and 15b) as the cause of SDH. Factor V Leiden was not detected and Co14A1 mutation was negative. Subsequently, the couple decided for termination of pregnancy based on the risk of significant neurological damage from iuMRI findings. However, this procedure was superseded by spontaneous labour when she presented with premature prelabour rupture of membranes and intrauterine death with a hydropic fetus at 28 weeks of gestation. A female baby of 1,805 $\mathrm{g}$ was delivered and her parents declined postmortem examination. The patient gave informed written consent for this case report.

\section{Methods}

A systematic review of fetal SDH was performed according to the PRISMA guideline. The systematic review was not registered. PubMed database was searched electronically on June 1, 2018, utilizing a combination of the relevant keywords for "fetal," "in utero," "intrauterine," "congenital," "prenatal," "subdural," "haemorrhage," and "haematoma." Manual check of the reference lists was performed. There was no restriction of language. The predefined criterion for study inclusion was ultrasonographic evidence of fetal SDH before delivery. The titles and the abstracts were screened for articles fulfilling the criteria. Full-text review of these articles was performed. Reference lists of relevant articles were searched manually for additional reports. Cases without prenatal imaging, SDH after delivery, or other sites of intracranial haemorrhage were ex- 

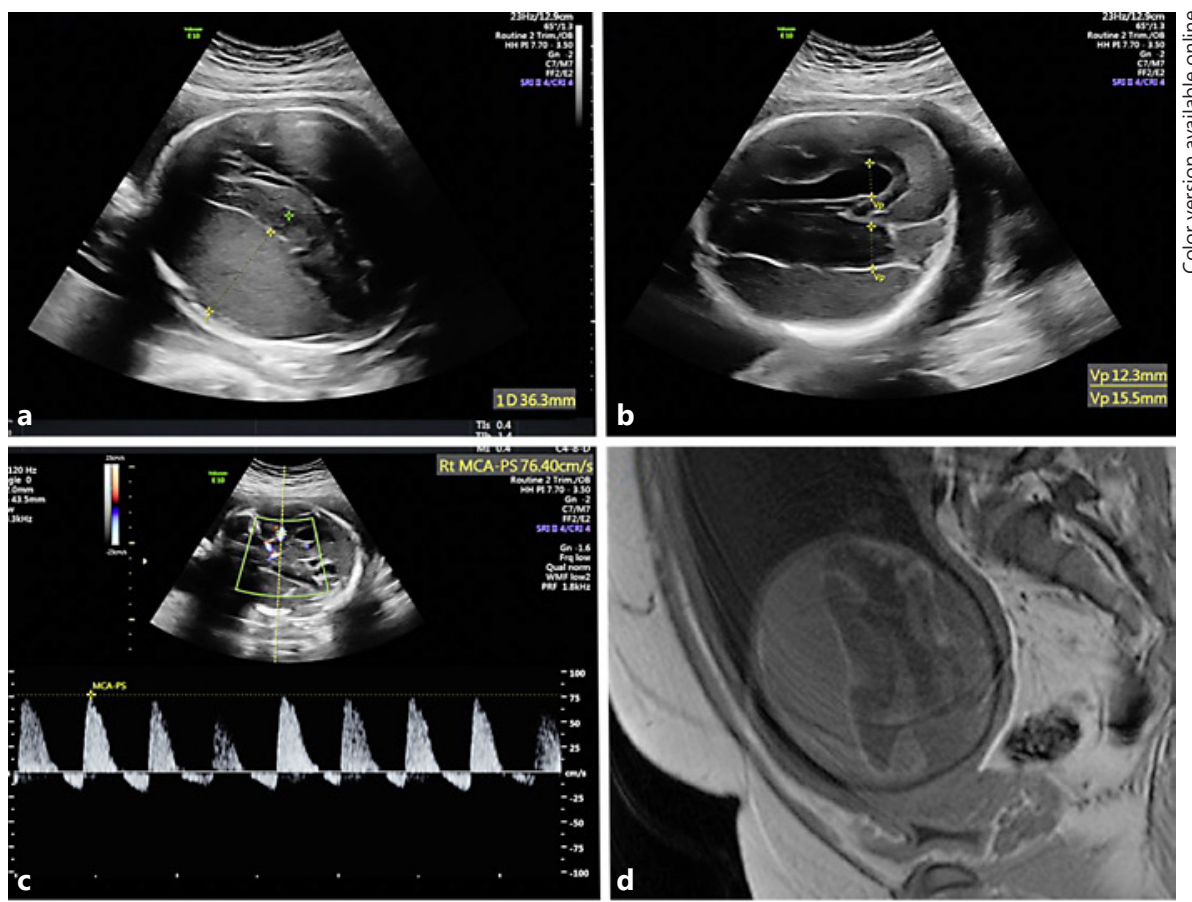

Fig. 1. a-c Fetal ultrasonography. a Rightsided fetal subdural haematoma causing midline shift to the left. b Bilateral ventriculomegaly. c Doppler of middle cerebral artery, reversed end diastolic flow with elevated peak systolic velocity. d-f In utero magnetic resonance imaging. T1-weighted (d) and T2-weighted imaging (e, f) showing fetal extra-axial haematoma and midline shift to the left with intraventricular haemorrhage as shown by the arrows. Cystic appearances of the left frontal lobe represent the areas of porencephaly. Bilateral cerebral infarctions were seen in diffusionweighted imaging $(\mathbf{g})$ showing areas with hyperintense signal involving the majority of both cerebral hemispheres (f). Apparent diffusion coefficient imaging showing areas with corresponding hypointense signal.
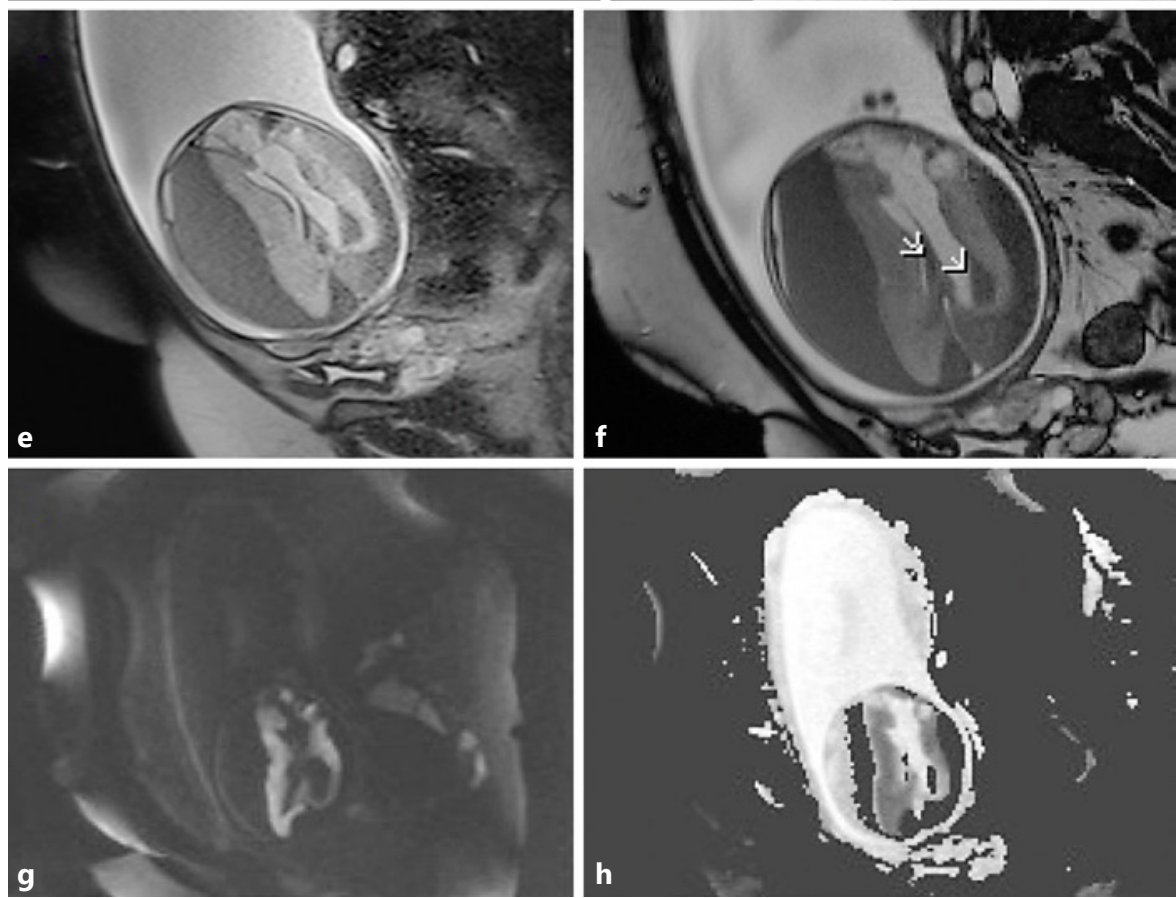

cluded. Two reviewers (K.W.C. and L.N.T.) performed the inclusion and exclusion of the articles independently, and any differences were resolved by a third reviewer (M.D.K.).

We extracted the following data from each report: the women's age, gravida and parity, gestational age at presentation, possible underlying aetiology for SDH, prenatal ultrasonographic features, utility of iuMRI, prenatal intervention, mode of delivery, neonatal treatment, and neonatal outcome. Quality assessment by the
Strengthening the Reporting of Observational Studies in Epidemiology checklist was not possible as only single case reports or small case series were identified and publication bias was noted. The primary outcome of the study was the neurological disability at last follow-up.

Data analysis was performed using Excel and GraphPad Prism version 6.00 for Windows (GraphPad Software, La Jolla, CA, USA). Results were presented as mean (standard deviation) or 
Fig. 2. Systematic literature search for fetal subdural haematoma.
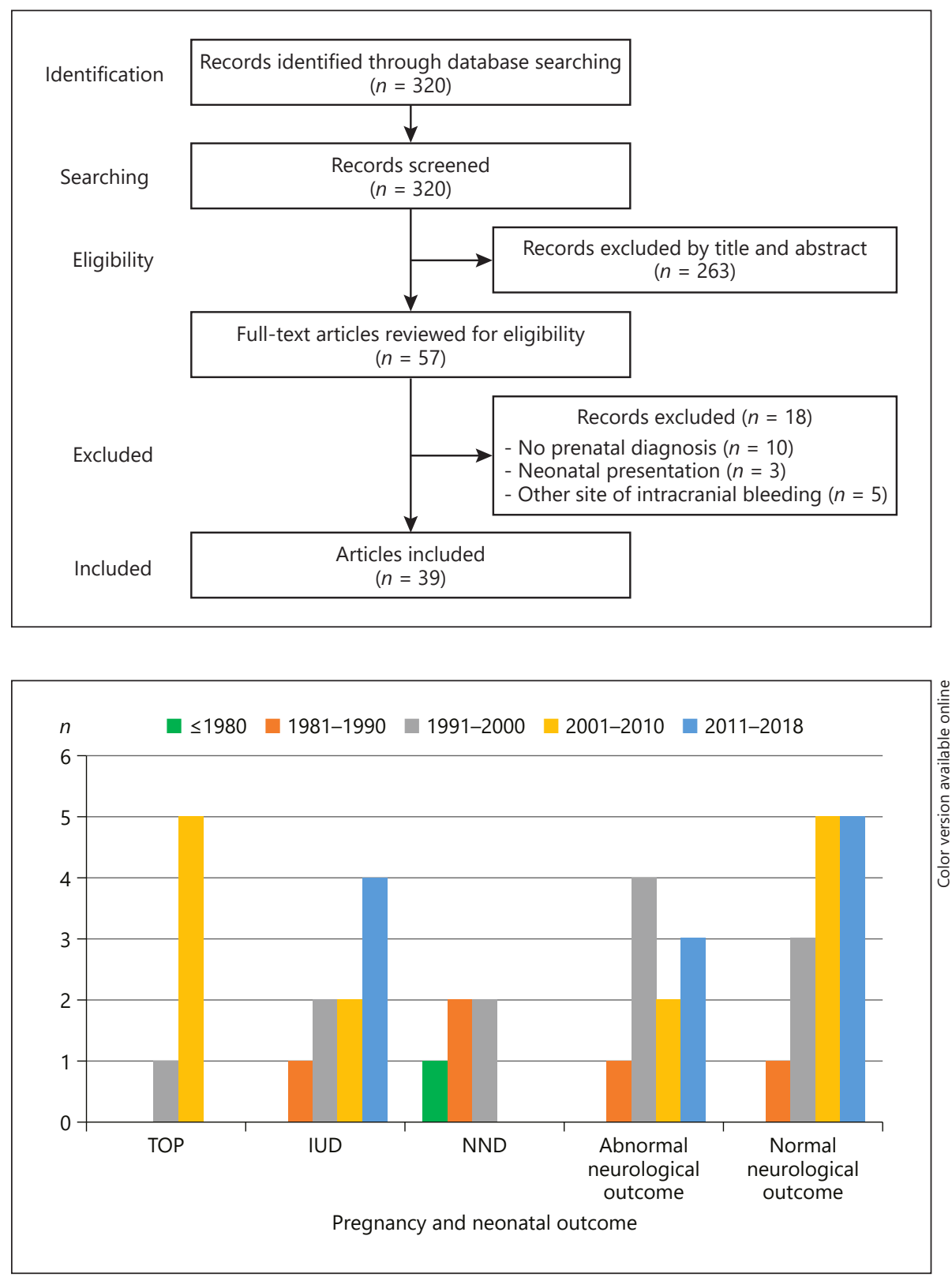

Fig. 3. Pregnancy and neonatal outcomes with reference to the year of publication. $n$ (\%). Difference of antenatal predictors between abnormal and normal neurology was investigated by $\chi^{2}$ test or Fisher exact test. Results were considered statistically significant when $p<0.05$.

\section{Results}

Figure 2 shows the literature identification and selection process. Table 1 summarises the basic demographics. A total of 45 cases were extracted ( 44 cases from 39 papers and our present case; online suppl. Table 1, see www. karger.com/doi/10.1159/000496202) [3, 5-42]. 78\% of cases were diagnosed prenatally after 24 weeks of gestation. Fetal sex was reported in 30 cases, of which $67 \%$ were male and $33 \%$ were female. iuMRI was not performed in 11 cases $(24 \%)$ because of sufficient ultrasonographic features to diagnose fetal SDH, and in 3 cases (7\%) because there were no antenatal suspicions of fetal SDH on ultrasonography. iuMRI was done in 16 cases (36\%) for abnormal cranial ultrasonographic findings but without antenatal ultrasonographic suspicions of fetal SDH prior to the iuMRI findings of SDH. In a further 12 cases (27\%), 
Table 1. Basic demographic of fetal SDH cases [3, 5-42]

\begin{tabular}{|c|c|c|}
\hline & $n$ & $\begin{array}{l}\text { Mean (SD), median (inter- } \\
\text { quartile), or } n(\%)\end{array}$ \\
\hline Maternal age, years & 38 & $29.8(5.9)$ \\
\hline Gravida & 26 & $2.7(2.4)$ \\
\hline Parity & 28 & $1.7(2.2)$ \\
\hline Gestational age at presentation, weeks & 45 & $29.2(4.3)$ \\
\hline Interval between prenatal diagnosis to delivery, weeks & 22 & $4.1(4.4)$ \\
\hline Suspected fetal SDH before MRI & 42 & $23(54.8)$ \\
\hline Mode of delivery (excluding stillbirth and termination of pregnancy) & 26 & \\
\hline Vaginal delivery & & $6(23.1)$ \\
\hline Caesarean delivery & & $20(76.9)$ \\
\hline Prenatal treatment & 8 & \\
\hline Maternal vitamin K supplementation & & $2(25.0)$ \\
\hline Switching from warfarin to heparin anticoagulant & & $2(25.0)$ \\
\hline Maternal betamethasone & & $1(12.5)$ \\
\hline Maternal weekly intravenous immunoglobulin & & $1(12.5)$ \\
\hline Intrauterine transfusion & & $2(25.0)$ \\
\hline Postnatal treatment & 26 & \\
\hline Drainage of haematoma & & $15(57.7)$ \\
\hline Red cell transfusion & & $4(15.4)$ \\
\hline Fresh frozen plasma & & $4(15.4)$ \\
\hline Platelet transfusion & & $2(7.7)$ \\
\hline Vitamin K supplement & & $3(11.5)$ \\
\hline Factor VIII concentrate & & $1(3.8)$ \\
\hline Factor IX concentrate & & $1(3.8)$ \\
\hline Prenatal or neonatal evidence of fetal anaemia & 45 & $11(24.4)$ \\
\hline Fetal sex & 30 & \\
\hline Male & & $20(66.6)$ \\
\hline Female & & $10(33.3)$ \\
\hline Number of abnormal prenatal ultrasonographic features & 41 & \\
\hline 5 & & $1(2.4)$ \\
\hline 4 & & $2(4.9)$ \\
\hline 3 & & $13(31.7)$ \\
\hline 2 & & $11(26.8)$ \\
\hline 1 & & $14(34.1)$ \\
\hline
\end{tabular}

SDH, subdural haematoma; MRI, magnetic resonance imaging

iuMRI confirmed the prenatal ultrasonographic findings of SDH. The remaining 3 cases did not have sufficient information regarding the utility of iuMRI $[6,24]$. Postnatal imaging or postmortem examination confirmed the antenatal diagnosis of fetal SDH in 39 cases (87\%), and in the remaining cases, the diagnosis was based solely on prenatal imaging.

\section{Ultrasonographic Features of Fetal SDH}

The prenatal ultrasonographic features of fetal SDH are listed in Table 2. Prenatal ultrasonographic features were intracranial echogenicity (42\%), lateral ventriculomegaly (38\%), presence of an intracranial mass (31\%), macrocephaly (24\%), midline deviation of cerebral falx (20\%), and an intracranial fluid collection (11\%). Further secondary features were noted including reversed diastolic flow in the middle cerebral artery (11\%), echogenic bowel (4\%), hydrops fetalis (2\%), and elevated middle cerebral artery peak systolic velocity (2\%) (all highly likely to be associated with fetal anaemia). In 6 cases of fetal $\mathrm{SDH}$, there were no details of prenatal ultrasonographic features $[3,22]$. One woman had normal prenatal ultrasonography [23]. Another woman did not receive prenatal ultrasonography and SDH was an incidental diagnosis following a computed tomography scan for abdominal trauma [24]. 39\% (16/41) had three or more prenatal ul- 
Fig. 4. Suggested clinical approach to fetal

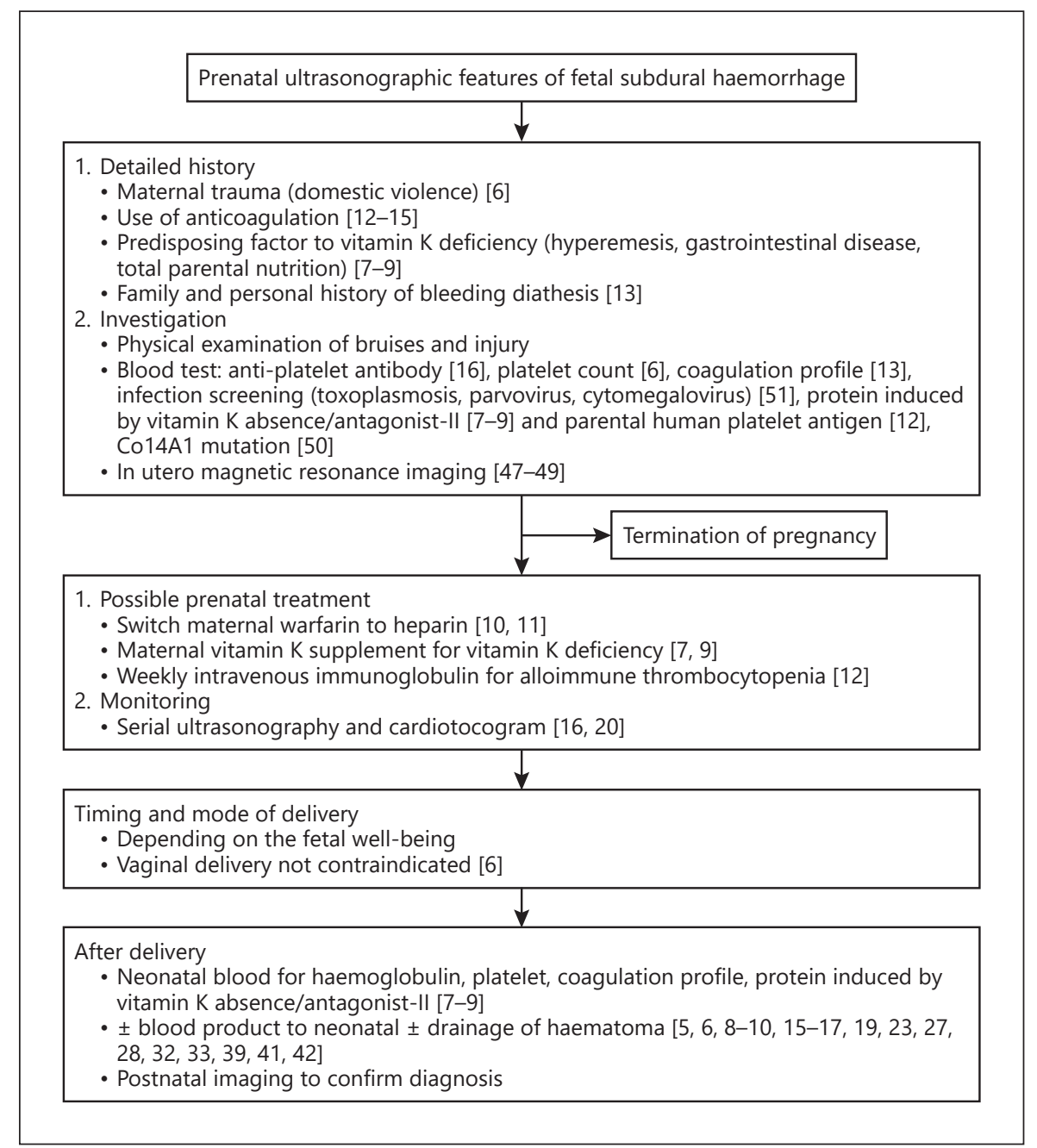
subdural haematoma.

trasonographic features (Table 1). Increased fetal head biometry was the only ultrasonographic feature in 3 cases (6\%, all cases published before 2000) and isolated ventriculomegaly was the only finding in 5 cases (11\%).

\section{Aetiology of SDH, Pregnancy, and Neonatal Outcome}

The possible aetiologies and fetal outcomes are listed in Table 3. 18\% had termination of pregnancy (8/45). In the remaining cases, $32 \%$ of fetuses with SDH had stillbirth (7/37) or neonatal death (5/37). 68\% of fetuses survived after fetal SDH (25/37). The underlying cause of fetal SDH was not identified in a significant number of cases $(21 / 45,47 \%)$. Maternal trauma $(6 / 45,13 \%)$ and use of warfarin $(5 / 45,11 \%)$ were the more common causes for SDH. The timing of postnatal neurological assessment was available in 18 cases. The median duration of followup was 10.5 months (ranging from 3 months to 7 years).
Table 2. Prenatal ultrasonographic features of fetal subdural haematoma

\begin{tabular}{lc}
\hline Ultrasonographic findings & $n(\%)$ \\
\hline Intracranial echogenicity & $19(42.2)$ \\
Ventriculomegaly & $17(37.8)$ \\
Intracranial mass & $14(31.1)$ \\
Increased fetal head measurement & $11(24.4)$ \\
Midline shift & $9(20.0)$ \\
Intracranial fluid collection & $5(11.1)$ \\
Reverse diastolic flow in the middle cerebral artery & $5(11.1)$ \\
Intraventricular haemorrhage & $4(8.9)$ \\
Echogenic bowel & $2(4.4)$ \\
Hydrops & $1(2.2)$ \\
Normal ultrasonography & $1(2.2)$ \\
Elevated middle cerebral artery peak systolic velocity & $1(2.2)$ \\
\hline
\end{tabular}


Table 3. Aetiology, pregnancy, and neonatal outcomes of 45 cases of fetal subdural haematoma

\begin{tabular}{|c|c|c|c|c|c|c|}
\hline & $n$ & TOP & IUD & NND & $\begin{array}{l}\text { Abnormal } \\
\text { neurological } \\
\text { outcome }\end{array}$ & $\begin{array}{l}\text { Normal } \\
\text { neurological } \\
\text { outcome }\end{array}$ \\
\hline Trauma & 6 & 0 & 1 & 0 & 1 & 4 \\
\hline Maternal use of warfarin & 5 & 2 & 0 & 1 & 0 & 2 \\
\hline Vitamin K deficiency & 3 & 0 & 0 & 0 & 1 & 2 \\
\hline Coagulopathy & 2 & 0 & 0 & $1^{\dagger}$ & 0 & 1 \\
\hline Autoimmune thrombocytopenia & 2 & 0 & 0 & 1 & 0 & 1 \\
\hline Alloimmune thrombocytopenia & $1^{\ddagger}$ & 0 & 0 & 0 & 0 & 0 \\
\hline Maternal use of low molecular weight heparin & 1 & 0 & 0 & 0 & 1 & 0 \\
\hline $\begin{array}{l}\text { In utero death of one monochorionic twin, } \\
\text { possible twin-to-twin transfusion }\end{array}$ & 1 & 1 & 0 & 0 & 0 & 0 \\
\hline
\end{tabular}

TOP, termination of pregnancy; IUD, intrauterine death; NNT, neonatal death. ${ }^{\dagger}$ One infant death at 7 months due to complication of congenital factor X deficiency. ${ }^{\ddagger}$ Neurological outcome not available at follow-up.

Table 4. Antenatal predictors of neurological status in survivors

$\begin{array}{lll}\begin{array}{l}\text { Abnormal neurological } \\ \text { outcome }(n=10)\end{array} & \begin{array}{l}\text { Normal neurological } \\ \text { outcome }(n=14)\end{array} & p \text { value }\end{array}$

\begin{tabular}{|c|c|c|c|}
\hline \multicolumn{4}{|l|}{ Underlying aetiology } \\
\hline No cause identified & $7(70 \%)$ & $3(21.43 \%)$ & 0.035 \\
\hline With underlying causes & $3(30 \%)$ & $11(78.57 \%)$ & \\
\hline Trauma & $1(10 \%)$ & $4(28.57 \%)$ & 0.358 \\
\hline Vitamin K deficiency & $1(10 \%)$ & $2(14.29 \%)$ & 1.000 \\
\hline Fetal vascular anomaly & $0(0 \%)$ & $1(7.14 \%)$ & 1.000 \\
\hline Coagulopathy & $0(0 \%)$ & $1(7.14 \%)$ & 1.000 \\
\hline Maternal use of low molecular weight heparin & $1(10 \%)$ & $0(0 \%)$ & 0.417 \\
\hline \multicolumn{4}{|l|}{ Ultrasonographic findings } \\
\hline Intracranial echogenicity & $3(30 \%)$ & $6(42.86 \%)$ & 0.679 \\
\hline Ventriculomegaly & $4(40 \%)$ & $5(35.71 \%)$ & 1.000 \\
\hline Intracranial mass & $1(10 \%)$ & $3(21.43 \%)$ & 0.615 \\
\hline Increased fetal head measurement & $4(40 \%)$ & $3(21.43 \%)$ & 0.393 \\
\hline Echogenic bowel & $0(0 \%)$ & $0(0 \%)$ & 1.000 \\
\hline Hydrops & $0(0 \%)$ & $1(7.14 \%)$ & 1.000 \\
\hline Elevated middle cerebral artery peak systolic velocity & $0(0 \%)$ & $0(0 \%)$ & 1.000 \\
\hline \multicolumn{4}{|l|}{ Ultrasonographic features, $n$} \\
\hline 5 & $0(0 \%)$ & $0(0 \%)$ & 1.000 \\
\hline 4 & $0(0 \%)$ & $0(0 \%)$ & 1.000 \\
\hline 3 & $3(30 \%)$ & $5(35.71 \%)$ & 1.000 \\
\hline 2 & $0(0 \%)$ & $2(14.29 \%)$ & 0.493 \\
\hline 1 & $5(50 \%)$ & $4(28.57 \%)$ & 0.403 \\
\hline
\end{tabular}


Amongst the 24 survivors with neurological outcome, 14 (58\%) had normal neurological outcome, while 10 (42\%) had a certain degree of neurological impairment. Antenatal predictive factors for neurological outcome in survivors are shown in Table 4. Fetal SDH with an identifiable underlying aetiology was the only factor associated with better neurological prognosis and a higher chance of having normal neurological outcome when compared to fetal $\mathrm{SDH}$ without a detectable underlying cause (78.5 vs. $21.4 \%, p=0.035)$. Figure 3 illustrates the pregnancy and neonatal outcomes with reference to the year of publication. Twenty-seven cases were published after 2000, there were no neonatal deaths, and an increasing number of cases with normal neurological outcome was observed. The number was too small for distribution analysis.

\section{Discussion}

Our pooled results suggested $32 \%$ of the fetuses with $\mathrm{SDH}$ would die either in utero or during the neonatal period. Normal neurological outcome was observed in 58\% of the survivors, whereas the remaining $42 \%$ had neurological morbidity. In contrast, earlier reports by Vergani et al. [1] and Akman and Cracco [5] have shown a higher risk of perinatal mortality $[1,5]$ and lower rate of normal neurological development [1]. The discrepancy could be explained by the improvement in neonatal care in the more recently reported cases, thus reducing neonatal mortality with increasingly normal neurological outcomes. In addition, Akman and Cracco [5] included 16 cases of stillbirth from one single case series [43], which retrospectively evaluated the potential underlying causes of stillbirth. Although the histological demonstration of haemosiderin in some cases might suggest in utero occurrence of $\mathrm{SDH}$, we have excluded these cases because these were postmortem findings without prenatal ultrasonography correlation, whereas the aim of this review was to focus on the management of antenatally detected fetal $\mathrm{SDH}$. In addition, all 16 cases of stillbirth in the single case series were Pacific Islanders [43], which may suggest some specific genetic/ethnic predisposition and the inclusion of these cases may affect the generalisation of our result. The finding of improved prognosis for fetal SDH with identified underlying aetiology compared to those without a detectable cause should be interpreted with caution. Detailed pre- and postnatal investigations were not done in many cases and this would affect our result significantly. Moreover, more advanced and up-to-date investigation regimes may be more readily available in centres with higher provisions in healthcare, which can improve the detection and management of SDH leading to improved neonatal outcomes. Nevertheless, optimisation of antenatal care and avoidance of trauma to reverse or reduce further bleeds may be contributing factors for better outcomes in cases with known bleeding tendencies.

The pathophysiology of atraumatic fetal SDH remains unknown and is extrapolated from SDH of shaken baby syndrome in infants $[44,45]$. The relatively higher brainto-fluid ratio in a fetal head may result in increased mobility of the fetal brain and thus the risk of severe bleeding when the fetal head is moved by external impact, causing stretching and tearing of the bridging veins in the subdural space without evidence of maternal trauma seen externally. As pregnancy progresses, the fetal head is closer to the wall of the uterus and maternal abdomen and therefore increases the chances of being affected by apparently minor direct trauma to the maternal abdomen. These may explain why the causes of fetal SDH cannot be identified in nearly half of the cases and the majority occurs in the third trimester. The presence of additional risk factors for fetal bleeding tendency may further increase the chance of fetal SDH.

The ultrasonographic appearances of the fetal SDH depend on the timing of ultrasonography since the initial bleed as well as the mass and pressure effects from the haematoma. An acute bleed is often echogenic on ultrasonography, whereas chronic SDH appears more translucent [46]. Mixed echogenicity on prenatal ultrasound can be visualized during the transitional stage due to the gradual breakdown of heamoglobin and liquefaction of the $\mathrm{SDH}$, or when acute bleeding occurs into the chronic $\mathrm{SDH}$. Significant mass effect could lead to midline shift or ventriculomegaly by aqueductal obstruction. The expanding volume from SDH may cause elevated intracranial pressure and increase in fetal head measurements as the sutures are patent during the fetal life. Echogenic bowel from intestinal bleeding could be an additional feature in pathology with generalized bleeding tendency (fetal thrombocytopenia or coagulopathy).

iuMRI is an important adjunct to ultrasonography to aid prognostic evaluation, parental counseling, and postnatal management [47-49]. Fetal SDH often occurs in the third trimester, when iuMRI has a higher diagnostic accuracy for cranial anomaly compared to ultrasonography, due to the deeply engaged fetal head and increased cranial ossification during this period $[47,48]$. In this review, we have found that iuMRI is potentially useful in at least $42 \%$ (19/45, without antenatal suspicions of fetal SDH by ultrasonography) of cases. 
The optimal management of fetal SDH remains controversial. A suggested clinical approach is outlined in Figure 4. A detailed history and blood test are vital to elucidate the underlying cause [6-9, 12-16, 47-51]. Termination of pregnancy is an option in view of the potential significant neurological deficit. However, women should be counseled about the complexity in predicting neonatal outcomes based solely on prenatal ultrasonographic findings. Green-Thompson and Moodley [22] reported a case of fetal SDH, bilateral intracerebral haemorrhage, and cortical atrophy with ventriculomegaly. The patient went into spontaneous labour before the discussion of termination of pregnancy and the infant had normal neurological assessment at postnatal follow-up [22]. Barozzino et al. [20] also reported spontaneous resolution of bilateral fetal subdural haematoma before delivery and favourable neonatal neurological outcome 9 months after delivery. If a woman opts for continuation of pregnancy, serial ultrasonographic monitoring is necessary for the progression of haemorrhage. Doppler studies of middle cerebral artery may reveal elevated peak systolic velocity, which indicates fetal anaemia due to SDH. Prenatal treatment would be possible if an aetiology is identified, such as maternal vitamin $\mathrm{K}$ supplementation in vitamin $\mathrm{K}$ deficiency $[7,9]$, switching maternal anticoagulation from warfarin to heparin $[10,11]$, and intravenous immunoglobulin in fetal alloimmune thrombocytopenia [12]. The role of fetal intervention (platelet or red cell transfusion) in SDH with fetal anaemia/thrombocytopenia is unclear. It should be balanced against the risks of preterm delivery, stillbirth, and fetal haemorrhage, especially in a fetus with potential thrombocytopenia/coagulopathy [51]. The optimal time and mode of delivery remain uncertain. The principle is to balance the risks of preterm delivery, stillbirth, and further fetal haemorrhage. The mode of delivery in the majority of cases reported is by Caesarean section, due to prematurity, fetal distress, and theoretical concerns of fetal intracranial bleeding during vaginal delivery. However, there is no strong evidence to suggest that Caesarean section can improve neonatal outcome in fetal SDH and therefore, both options should be considered after adequate parental counseling. After delivery, neonatal blood test is required to rule out anaemia, thrombocytopenia, or disseminated intravascular coagulation secondary to fetal SDH.

This systematic review had several limitations. Only case reports and case series were identified with possible consequent publication bias. The inconsistencies in reporting antenatal investigations, prenatal ultrasonographic features, and neurological outcomes affect the in- terpretation of our results. The sample size is also too small to allow meaningful subgroup analysis. However, given the rarity of this topic and difficulties in conducting prospective studies, we have reported the pooled outcomes of fetal SDH and proposed a clinical algorithm based on the best presently available evidence.

\section{Conclusions}

Stillbirth and neonatal death occurred in $32 \%$ of fetal SDH. 58\% of survivors had normal neurological outcomes, whereas the remaining $42 \%$ may suffer from neurological impairment. Detailed prenatal investigation is crucial to elucidate the underlying aetiology, which may be associated with a better prognosis.

\section{Acknowledgement}

We thank Weilan Wang, The University of Hong Kong, for statistical analysis.

\section{Statement of Ethics}

The authors have no ethical conflicts to disclose.

\section{Disclosure Statement}

The authors have no conflicts of interest to declare.

\section{Funding Sources}

No funding to declare.

\section{Author Contributions}

K.W.C, L.N.T, and M.T.Y.S. prepared the manuscript. All authors contributed to the conception of the study and were involved in amendment and the approval of the final manuscript. 


\section{References}

1 Vergani P, Strobelt N, Locatelli A, Paterlini G, Tagliabue P, Parravicini E, et al. Clinical significance of fetal intracranial hemorrhage. Am J Obstet Gynecol. 1996 Sep;175(3 Pt 1): 536-43.

2 Kutuk MS, Yikilmaz A, Ozgun MT, Dolanbay M, Canpolat M, Uludag S, et al. Prenatal diagnosis and postnatal outcome of fetal intracranial hemorrhage. Childs Nerv Syst. 2014 Mar; 30(3):411-8.

3 Abdelkader MA, Ramadan W, Gabr AA, Kamel A, Abdelrahman RW. Fetal intracranial hemorrhage: sonographic criteria and merits of prenatal diagnosis. J Matern Fetal Neonatal Med. 2017 Sep;30(18):2250-6.

4 Whitby EH, Griffiths PD, Rutter S, Smith MF, Sprigg A, Ohadike P, et al. Frequency and natural history of subdural haemorrhages in babies and relation to obstetric factors. Lancet. 2004 Mar;363(9412):846-51.

5 Akman CI, Cracco J. Intrauterine subdural hemorrhage. Dev Med Child Neurol. 2000 Dec;42(12):843-6.

6 Ghi T, Simonazzi G, Perolo A, Savelli L, Sandri F, Bernardi B, et al. Outcome of antenatally diagnosed intracranial hemorrhage: case series and review of the literature. Ultrasound Obstet Gynecol. 2003 Aug;22(2):121-30.

7 Hirose M, Akiyama M, Takakura K, Noda Y. Active Crohn disease with maternal vitamin $\mathrm{K}$ deficiency and fetal subdural hematoma. Obstet Gynecol. 2001 Nov;98(5 Pt 2):919-21.

8 Sakai M, Yoneda S, Sasaki Y, Saito S. Maternal total parenteral nutrition and fetal subdural hematoma. Obstet Gynecol. 2003 May;101(5 Pt 2):1142-4.

9 Sasaki Y, Kikuchi A, Suga Y, Haba G, Kanasugi T, Isurugi C, et al. Progressive Fetal Subdural Hematoma Associated With Maternal Vitamin K Deficiency: Prenatal Diagnosis and Neurologically Favorable Prognosis. J Ultrasound Med. 2017 Sep;36(9):1961-3.

10 Fujiwara K, Aoki S, Kurasawa K, Okuda M, Takahashi T, Hirahara F. Warfarin-associated fetal intracranial subdural hematoma: a case report. Clin Case Rep. 2014 Jun;2(3) 108-11.

11 Matsuda Y, Hashiguchi K, Akizawa Y, Saito $\mathrm{R}$, Ohta H. A case of fetal subdural hematoma at 31 weeks of gestation in a woman on warfarin therapy after Cabrol's operation. Fetal Diagn Ther. 2003 Jul-Aug;18(4):252-4.

12 Oswal K, Agarwal A. Warfarin-induced fetal intracranial subdural hematoma. J Clin Ultrasound. 2008 Sep;36(7):451-3.

13 Lee HC, Cho SY, Lee HJ, Kim CJ, Park JS, Ch JG. Warfarin-associated fetal intracranial hemorrhage: a case report. J Korean Med Sci. 2003 Oct;18(5):764-7.

14 Robinson MJ, Cameron MD, Smith MF Ayers AB. Fetal subdural haemorrhages presenting as hydrocephalus. BMJ. 1980 Jul; 281(6232):35.
15 Bauder F, Beinder E, Arlettaz R, Albisetti M, Boltshauser E, Gessler P. Intrauterine subdural hemorrhage in a preterm neonate possibly associated with maternal low-molecular weight heparin treatment. J Perinatol. 2009 Jul;29(7):521-3.

16 de Spirlet M, Goffinet F, Philippe HJ, Bailly M, Couderc S, Nisand I. Prenatal diagnosis of a subdural hematoma associated with reverse flow in the middle cerebral artery: case report and literature review. Ultrasound Obstet Gynecol. 2000 Jul;16(1):72-6.

17 Rios LT, Araujo Júnior E, Nardozza LM, Haratz KK, Moron AF, Martins MG. Hidden maternal autoimmune thrombocytopenia complicated by fetal subdural hematoma-case report and review of the literature. Childs Nerv Syst. 2012 Jul;28(7):1113-6.

18 Meagher SE, Walker SP, Choong S. Mid-trimester fetal subdural hemorrhage: prenatal diagnosis. Ultrasound Obstet Gynecol. 2002 Sep;20(3):296-8.

19 de Sousa C, Clark T, Bradshaw A. Antenatally diagnosed subdural haemorrhage in congenital factor X deficiency. Arch Dis Child. 1988 Oct;63(10 Spec No):1168-70.

20 Barozzino T, Sgro M, Toi A, Akouri H, Wilson S, Yeo E, et al. Fetal bilateral subdural haemorrhages. Prenatal diagnosis and spontaneous resolution by time of delivery. Prenat Diagn. 1998 May;18(5):496-503.

21 Matsushita H, Harada A, Sato T, Kurabayashi T. Fetal intracranial injuries following motor vehicle accidents with airbag deployment. J Obstet Gynaecol Res. 2014 Feb;40(2):599602.

22 Green-Thompson R, Moodley J. In-utero intracranial haemorrhage probably secondary to domestic violence: case report and literature review. J Obstet Gynaecol. 2005 Nov; 25(8):816-8.

23 Piastra M, Pietrini D, Massimi L, Caldarelli M, De Luca D, Del Lungo LM, et al. Severe subdural hemorrhage due to minimal prenatal trauma. J Neurosurg Pediatr. 2009 Dec; 4(6):543-6.

24 Zeina AR, Kessel B, Mahamid A, Gazmawi J, Shrim A, Nachtigal A, et al. Computed tomographic diagnosis of traumatic fetal subdural hematoma. Emerg Radiol. 2013 Apr;20(2): 169-72.

25 Paladini D, Sglavo G, Quarantelli M, D'armiento MR, Martinelli P, Salvatore M. Large infratentorial subdural hemorrhage diagnosed by ultrasound and MRI in a secondtrimester fetus. Ultrasound Obstet Gynecol. 2005 Dec;26(7):789-91.

26 Strigini FA, Nardini V, Carmignani A, Valleriani AM. Second-trimester diagnosis of intracranial vascular anomalies in a fetus with subdural hemorrhage. Prenat Diagn. 2004 Jan;24(1):31-4.
27 Sohda S, Hamada H, Takanami Y, Kubo T. Prenatal diagnosis of fetal subdural haematomas. Br J Obstet Gynaecol. 1996 Jan;103(1) 89-90.

28 Hanigan WC, Ali MB, Cusack TJ, Miller TC, Shah JJ. Diagnosis of subdural hemorrhage in utero. Case report. J Neurosurg. 1985 Dec 63(6):977-9.

29 Kawabata I, Imai A, Tamaya T. Antenatal subdural hemorrhage causing fetal death before labor. Int J Gynaecol Obstet. 1993 Oct; 43(1):57-60.

30 Catanzarite VA, Schrimmer DB, Maida C, Mendoza A. Prenatal sonographic diagnosis of intracranial haemorrhage: report of a case with a sinusoidal fetal heart rate tracing, and review of the literature. Prenat Diagn. 1995 Mar;15(3):229-35.

31 Gunn TR, Mok PM, Becroft DM. Subdural hemorrhage in utero. Pediatrics. 1985 Oct; 76(4):605-10.

32 Nogueira GJ. Chronic subdural hematoma in utero. Report of a case with survival after treatment. Childs Nerv Syst. 1992 Dec;8(8): 462-4.

33 Mateos F, Esteban J, Ramos JT, Martín-Puerto MJ, Miralles M, Ozaita G, et al. Fetal subdural hematoma: diagnosis in utero. Case report. Pediatr Neurosci. 1987;13(3):125-8.

34 Pilalis A, Daskalakis G, Papantoniou N, Mesogitis S, Antsaklis A. Prenatal diagnosis of atraumatic fetal subdural hematoma. Am J Obstet Gynecol. 2003 Sep;189(3):882-3.

35 Batukan C, Holzgreve W, Bubl R, Visca E, Radü EW, Tercanli S. Prenatal diagnosis of an infratentorial subdural hemorrhage: case report. Ultrasound Obstet Gynecol. 2002 Apr 19(4):407-9.

36 Demir RH, Gleicher N, Myers SA. Atraumatic antepartum subdural hematoma causing fetal death. Am J Obstet Gynecol. 1989 Mar 160(3):619-20.

37 Rotmensch S, Grannum PA, Nores JA, Hall C, Keller MS, McCarthy S, et al. In utero diagnosis and management of fetal subdural hematoma. Am J Obstet Gynecol. 1991 May;164(5 Pt 1):1246-8.

38 Saadi H, Banani A. [Fetal subdural hematoma]. Pan Afr Med J. 2013 May;15:17.

39 Breningstall GN, Patterson RJ. Intrauterine subdural hematoma. Pediatr Radiol. 2000 Sep;30(9):630-1.

40 Ben-Chetrit A, Anteby E, Lavy Y, Zacut D, Yagel S. Increased middle cerebral artery blood flow impedance in fetal subdural hematoma. Ultrasound Obstet Gynecol. 1991 Sep; 1(5):357-8

41 Green PM, Wilson H, Romaniuk C, May P, Welch CR. Idiopathic intracranial haemorrhage in the fetus. Fetal Diagn Ther. 1999 SepOct;14(5):275-8

42 Lafont M, Lamarque M, Daussac E. [Favorable outcome of a subdural hematoma diagnosed in utero]. Arch Pediatr. 1999 Sep;6(9): 962-5. 
43 Gunn TR, Becroft DM. Unexplained intracranial haemorrhage in utero: the battered fetus? Aust N Z J Obstet Gynaecol. 1984 Feb;24(1): $17-22$.

44 Duhaime AC, Gennarelli TA, Thibault LE, Bruce DA, Margulies SS, Wiser R. The shaken baby syndrome. A clinical, pathological, and biomechanical study. J Neurosurg. 1987 Mar; 66(3):409-15.

45 Brown JK, Minns RA. Non-accidental head injury, with particular reference to whiplash shaking injury and medico-legal aspects. Dev Med Child Neurol. 1993 Oct;35(10):849-69.
46 Lam AH, Cruz GB. Ultrasound evaluation of subdural haematoma. Australas Radiol. 1991 Nov;35(4):330-2.

47 Griffiths PD, Bradburn M, Campbell MJ, Cooper CL, Graham R, Jarvis D, et al.; MERIDIAN collaborative group. Use of MRI in the diagnosis of fetal brain abnormalities in utero (MERIDIAN): a multicentre, prospective cohort study. Lancet. 2017 Feb; 389(10068):538-46.

48 Griffiths PD, Brackley K, Bradburn M, Connolly DJ, Gawne-Cain ML, Griffiths DI, et al. Anatomical subgroup analysis of the MERIDIAN cohort: ventriculomegaly. Ultrasound Obstet Gynecol. 2017 Dec;50(6):736-44.
49 Sanapo L, Whitehead MT, Bulas DI, Ahmadzia HK, Pesacreta L, Chang T, et al. Fetal intracranial hemorrhage: role of fetal MRI. Prenat Diagn. 2017 Aug;37(8):827-36.

50 Gould DB, Phalan FC, Breedveld GJ, van Mil SE, Smith RS, Schimenti JC, et al. Mutations in Col4al cause perinatal cerebral hemorrhage and porencephaly. Science. 2005 May; 308(5725):1167-71.

51 Espinoza JP, Caradeux J, Norwitz ER, Illanes SE. Fetal and neonatal alloimmune thrombocytopenia. Rev Obstet Gynecol. 2013; 6(1):e15-21. 